\title{
Enfance en Difficulté: Représentations des Enseignants Franco-Ontariens à l'Égard des Plans d'Enseignement Individualisés
}

\author{
Professeur Yvon Gauthier
}

Université Laurentienne

\begin{abstract}
Résumé
Cet article traite des représentations du personnel enseignant à propos des plans d'enseignement individualisés (PEI) destinés aux enfants en difficulté. L'intention est de cerner le lien entre le point de vue de ces intervenants et certaines de leurs caractéristiques personnelles et professionnelles. Une enquête a été menée auprès d'un échantillon du personnel enseignant franco-ontarien oeuvrant dans trois cycles d'enseignement différents. Les résultats laissent entrevoir, entre autres, que les représentations que se font les enseignants des PEI sont influencées par leur ancrage professionnel.
\end{abstract}

\section{Introduction}

Depuis 1998, et aux termes du Règlement 181/98 de la Loi de l'éducation de l'Ontario intitulée «Identification et placement des élèves en difficulté », un plan d'intervention personnalisé doit être élaboré pour tous les élèves atteints d'une anomalie. L'élaboration du

Yvon Gauthier est professeur titulaire à l'Écoles des sciences de l'éducation à l'Université Laurentienne, Sudbury (ON).

* Ce texte est dédié à feu Pounthioun Diallo 
plan est d'abord précédée d'une évaluation formelle de l'élève par un comité d'identification de placement et de révision du conseil scolaire. Les informations pertinentes au sujet de l'élève sont par la suite transmises à l'équipe qui prépare le PEI de l'élève. Cette équipe se compose de personnes qui connaissent et qui travaillent avec lui, notamment le personnel enseignant et toutes autres personnes qui ont la responsabilité de répondre aux besoins particuliers de l'élève en question.

Le guide du PEI du ministère de l'éducation, « Normes pour l'élaboration, la planification des programmes et la mise en oeuvre (2004) » destiné au personnel enseignant et les intervenants en enfance en difficulté pour la préparation d'un PEI, définit ce document comme « un plan écrit décrivant le programme d'enseignement et/ou les services à l'enfance en difficulté requis par l'élève, fondé sur une évaluation globale des points forts et des besoins de l'élève, c'est-à-dire les points forts et les besoins qui ont une incidence sur la capacité de l'élève d'apprendre et de démontrer son apprentissage...p.7 ». Suite à l'élaboration du PEI, le titulaire de l'élève a la responsabilité de mettre en pratique le plan d'intervention individualisé préparé pour son élève en difficulté. Ce plan, qui comprend, entre autres, des informations sur les attentes d'apprentissage et des stratégies pédagogiques spécifiques à l'élève, doit être révisé régulièrement afin de vérifier son efficacité. La mise en pratique du plan ainsi que sa révision représentent une tâche supplémentaire pour les enseignants, ce qui nous incite à nous demander ce que ces derniers pensent des PEI et de l'obligation qu'ils ont à en tenir compte dans leur quotidien.

\section{Problématique}

Depuis que la préparation d'un PEI est devenue obligatoire dans le système d'éducation ontarien, il y a eu énormément de controverse quant à son utilité pour l'enseignant. Dans les milieux scolaires, il est « commun » de qualifier cette pratique de « cancer pédagogique » ou de « tâche administrative qui contribue très peu à l'éducation des 
Yves Gauthier

enfants en difficulté ». Bien que cet état d'esprit soit documenté aux Etats-Unis (Bateman, 1995), il reste à mieux comprendre ses diverses dimensions en Ontario. Nous voulons donc apporter un éclairage sur cette question en vérifiant les représentations du personnel enseignant à l'égard du PEI. Quels facteurs influencent les représentations des enseignants? Qu'en est-il des stratégies proposées? Répondons-nous aux besoins des élèves en difficulté en utilisant un PEI ? Ce sont là autant de questions auxquelles nous avons tenté d'apporter quelques éléments de réponses dans la présente recherche. Notre hypothèse est que les représentations que se font les enseignants du PEI sont davantage influencées par leur expérience scolaire et le niveau d'enseignement que par des caractéristiques personnelles comme l'âge ou le sexe. Ainsi, le fait d'œuvrer auprès d'élèves d'âges variés et à des niveaux de scolarité différents agirait plus directement sur les représentations que les enseignants se font du PEI.

\section{Recension des écrits}

Les débats traitant de l'éducation des enfants en difficulté soulèvent des préoccupations très variées selon les pays, et ce, tant du côté des objets de recherche que des perspectives et des méthodologies qu'adoptent les chercheurs. Alors qu'en France et au Royaume-Uni les chercheurs en sont encore aux discussions sur l'inclusion (Armstrong, 2003) et sur des questions très générales portant sur l'enjeu de la présence des situations de handicaps au sein de l'institution scolaire (voir le numéro spécial que la Revue française de pédagogie consacre à ce sujet, en 2001), une orientation plus pragmatique a cours ailleurs dans le monde. Par exemple, en Nouvelle-Zélande, plusieurs travaux se penchent sur le processus qui sous-tend la conception du PEI, notamment ceux de Thomson et Rowan (1995). Nous avons déjà indiqué dans une étude récente (Gauthier et Diallo, 2004) que les gouvernements encadraient avec beaucoup de minutie le processus du PEI et que la rédaction de ce dernier était devenue une pratique courante en Amérique du Nord.

Dans la recherche comme dans la pratique, nous vivons toujours dans la période « infantile» des PEI (Bateson, 1995). Aux 
États-Unis, où la recherche sur l'enfance en difficulté est plus abondante, les thèmes traités varient peu. Les orientations privilégiées concernent surtout à l'élaboration du PEI en lien avec les aspects légaux ou pédagogiques (Drasgow, Yell et Robinson, 2001; Smith, 2000; Bateman et Herr, 2003; Bateman et Linden, 1998). Toutefois, nous y retrouvons aussi un intérêt pour l'évolution des PEI en lien avec la loi (Smith, 1990), pour l'implication des parents (Smith, 2001), pour le virage technologique dans la conception des PEI (Smith et Kortering, 2001), pour l'analyse des PEI selon les types de handicaps (Smith, 1990, Smith et Simpson, 1989), ainsi que pour l'art et les PEI (Di Maria, 1992). Au Canada, nous notons également une certaine préoccupation pour la démarche pédagogique ainsi que pour les composantes que doit contenir le PEI (Hartwick et Blattenberger, 1986; Goupil, 1991, 1997, 2004). Les auteurs québécois ont davantage prêté attention à la question de l'évaluation (Côté, Pilon, Dufour, et Tremblay, 1989) et à la façon de mieux connaître l'élève à travers le processus du PEI (Landry, 1990).

En Ontario, Gauthier (2004) a présenté une étude comparant, entre autres, les stratégies d'apprentissage des élèves surdoués à ceux de enfants en troubles d'apprentissage. Le ministère de l'Éducation et de la Formation (MÉFO) a vérifié si la mise en œuvre du PEI était conforme aux normes de l'éducation de la province. Cet examen des PEI a donné suite d'ailleurs à quelques modifications au nouveau guide du PEI (2004) pour la préparation de ceux-ci. Il s'agit ici des deux seules études sur le sujet en Ontario français.

L'accent mis sur les aspects pédagogiques du PEI est important puisqu'il nous renseigne sur la recherche des voies visant à mieux répondre aux besoins des élèves. Toutefois, il laisse dans l'ombre les attitudes et les conceptions des enseignants qui sont loin d'être négligeables dans la mise en œuvre des PEI. Cet aspect est peu traité dans les études. La recherche de Thomson et Rowan (1995), qui s'intéressent en partie sur les perceptions et représentations d'enseignants et de parents néo-zélandais sur le lien entre le PEI et l'éducation des enfants à besoins spéciaux, est l'une des rares études dans le domaine.

La recension des écrits nous laisse croire que les connaissances disponibles au sujet du PEI résultent parfois d'études 
empiriques, alors qu'en réalité elles demeurent encore largement théoriques. Ce sont les descriptions qui dominent à partir de points de vue traduisant la vision idéale que les auteurs ont des PEI et de leur utilisation auprès des élèves, ainsi que le montre, par exemple, l'étude historique de Smith (1990) dans le contexte américain. Par ailleurs, il apparaît que la plupart des études sur le PEI sont dépourvues d'assises théoriques explicites, ce qui limite généralement la fécondité des résultats.

\section{Cadre théorique}

L'hypothèse de cette recherche est formulée sur la base de la théorie des représentations sociales (Moscovici, 1961, 1989). Notre perspective est d'établir le rapport des enseignants au PEI comme le résultat non pas d'une expérience subjective mais d'une construction idéologique collective du groupe professionnel du personnel enseignant. La théorie des représentations sociales s'avère éclairante à cet égard. Par exemple, Jodelet (1989) conçoit une représentation sociale comme étant une forme de connaissance socialement élaborée et partagée, ayant une visée pratique et concourant à la construction d'une réalité commune à un ensemble social donné. Si elles influencent les conduites et la communication des groupes humains et de leurs membres, les représentations sont elles-mêmes façonnées par les rôles et l'expérience des acteurs sociaux (Doise et Palmonari, 1986). Parmi les processus qui participent à la formation et au fonctionnement des représentations sociales, les théoriciens se réfèrent, entre autres, à la notion d' " ancrage ». Cet ancrage permet de comprendre les catégories nouvelles porteuses d'une information plus ancienne, établies et partagées au sein du groupe. Le PEI, comme mesure pédagogique et légale relativement nouvelle dans le champ scolaire, pourrait bien se comprendre dans le rapport que le personnel enseignant entretient avec lui, à la lumière des représentations sociales.

\section{Méthodologie}

Les données de l'étude proviennent entièrement d'une enquête 
par questionnaire auprès du personnel enseignant d'écoles francoontariennes. Deux étapes ont marqué la conception de l'instrument de recherche. La première a consisté à sonder le pouls d'enseignants au sujet des PEI afin de bien cibler les questions de recherche. Pour ce faire, quatre groupes focus ont été formés: deux dans la région du moyen nord de l'Ontario, et deux dans la région de l'est de la province. Le but était d'avoir un échange libre avec les participants et participantes pour circonscrire les représentations les plus souvent évoquées dans les milieux scolaires au sujet du contenu des PEI. Au total, vingt membres du personnel enseignant des régions mentionnées ont participé à l'exercice.

La deuxième étape a mené à l'élaboration d'un questionnaire faisant appel à une échelle Likert avec des niveaux allant de 1 à 5: le chiffre « $1 »$ signifie que vous ne vous adonnez jamais à l'activité qui est désignée par l'énoncé; le chiffre « 5 » signifie que vous y adonnez très souvent; les chiffres « 2 » à « 4 » correspondent à des positions intermédiaires. Le questionnaire a été construit en tenant compte des points de vue exprimés par les enseignants lors des groupes focus. Il comprend cinq variables à expliquer: 1) le lien entre l'information contenue dans le PEI et les besoins des élèves, 2) le caractère pratique des stratégies d'enseignements proposées dans le PEI, 3) la fonction attribuée au PEI pour mieux connaître les élèves, 4) l'utilité pédagogique du PEI et 5) la pertinence des attentes des élèves dans le PEI.

Une question ouverte permettait aux répondants de partager d'autres commentaires au sujet des PEI. Les questions d'âge, sexe, années d'expérience, niveau d'enseignement et le nombre de cours suivis en enfance en difficulté ont servi de variables indépendantes.

\section{Échantillon}

Parmi les douze conseils scolaires francophones de la province de l'Ontario, huit ont accepté de participer à cette recherche qui, aux dires de plusieurs directeurs suscitait énormément d'intérêt dans les milieux scolaires. Cinquante questionnaires ont été acheminés directement aux personnes ressources de l'enfance en difficulté de 
chaque conseil, qui les ont ensuite distribués au personnel enseignant de différentes écoles. En tout, des 400 questionnaires distribués, nous en avons reçu 329 pour un taux de participation de $82,25 \%$, ce qui témoigne de l'énorme intérêt porté à l'égard des PEI dans les écoles françaises de l'Ontario.

Les répondants proviennent de tous les coins de la province puisque huit conseils scolaires ont participé à la recherche. On compte 251 femmes $(76,3 \%)$ par rapport à 78 hommes $(21,6 \%)$ et l'âge moyen est de 37,4\% ans. De ce nombre, 143 participants enseignent au primaire-moyen (47,5 pour cent), 79 au primaire-intermédiaire $(23,9$ pour cent) et 86 ( 28,6 pour cent) sont au niveau intermédiairesecondaire. En moyenne, le personnel enseignant a 12,14 années d'expérience. De plus, 167 (50,8\%) indiquent ne jamais avoir suivi de cours de qualification additionnelle en enfance en difficulté, 64 ont suivi la partie $1(19,5$ pour cent), $20(6,1 \%)$ et 78 ( 23,7 pour cent) ont suivi la partie 3; c'est-à-dire qu'ils sont spécialistes dans ce domaine.

\section{Résultats}

Les tableaux 1 et 2 présentent des données de base au sujet de l'utilisation des PEI par le personnel enseignant qui a participé à cette recherche. Cette distribution de fréquences présente également les données des répondants pour toutes les questions du questionnaire. Les tableaux nous permettent ainsi d'avoir une vue d'ensemble sur les opinions du personnel enseignant concernant les PEI. Selon l'échelle Likert que nous avons utilisée, le « $1 »$ signifie que les répondants et répondantes ne s'adonnent jamais à l'activité désignée, tandis que le « 5 » indique qu'ils s'y adonnent souvent.

Ces tableaux tracent un aperçu des opinions du personnel enseignant en lien avec la question des plans d'enseignement individualisés. Nous notons, par exemple, que les répondants n'entretiennent pas d'opinions très positives au sujet du PEI en tant qu'outil pédagogique ( $\mathrm{X}=3,65$ selon l'échelle Likert). Dans le même ordre d'idées on remarque que les répondants ont tendance à être d'accord avec l'énoncé selon lequel ils consultent les PEI pour mieux connaître les élèves $(X=4,00)$. 


\begin{tabular}{|l|l|l|l|l|l|l|}
\hline & $\begin{array}{l}\text { Utilise le } \\
\text { PEI }\end{array}$ & $\begin{array}{l}\text { Consulte } \\
\text { le PEI }\end{array}$ & $\begin{array}{l}\text { Attentes } \\
\text { sont } \\
\text { pertinentes }\end{array}$ & $\begin{array}{l}\text { Inf. reflète } \\
\text { le besoin } \\
\text { des élèves }\end{array}$ & $\begin{array}{l}\text { Outil } \\
\text { péd. } \\
\text { utile }\end{array}$ & $\begin{array}{l}\text { Diagnostic } \\
\text { est précis }\end{array}$ \\
\hline Moyenne & 3,93 & 4,00 & 3,95 & 4,06 & 3,80 & 3,90 \\
\hline Médiane & 4,00 & 4,00 & 4,00 & 4,00 & 4,00 & 4,00 \\
\hline Mode & 5 & 5 & 5 & 5 & 4 & 4 \\
\hline $\begin{array}{l}\text { Déviation } \\
\text { Std. }\end{array}$ & 1,102 & 1,147 & 1,111 & 1,043 & 1,095 & .987 \\
\hline
\end{tabular}

Tableau 1. Vue d'ensemble des opinions du personnel enseignant au sujet des PEI

\begin{tabular}{|l|l|l|l|l|l|l|}
\hline & $\begin{array}{l}\text { Stratégies } \\
\text { sont } \\
\text { pratiques }\end{array}$ & $\begin{array}{l}\text { Connais- } \\
\text { sances } \\
\text { des } \\
\text { anomalies } \\
\text { sont } \\
\text { adéquates }\end{array}$ & $\begin{array}{l}\text { Revoir } \\
\text { les } \\
\text { normes } \\
\text { du PEI }\end{array}$ & $\begin{array}{l}\text { PEI est } \\
\text { un outil } \\
\text { péd. }\end{array}$ & $\begin{array}{l}\text { PEI est } \\
\text { une } \\
\text { tâche } \\
\text { admin- } \\
\text { istrative }\end{array}$ & $\begin{array}{l}\text { Éducation } \\
\text { répond } \\
\text { aux } \\
\text { besoins } \\
\text { des élèves }\end{array}$ \\
\hline Moyenne & 3,69 & 3,71 & 3,89 & 3,65 & 3,61 & 4,06 \\
\hline Médiane & 4,00 & 4,00 & 4,00 & 4,00 & 4,00 & 4,00 \\
\hline Mode & 4 & 4 & 5 & 3 & 5 & 5 \\
\hline $\begin{array}{l}\text { Déviation } \\
\text { Std. }\end{array}$ & 1,067 & .983 & 1,062 & 1,137 & 1,247 & 1,043 \\
\hline
\end{tabular}

Tableau 2: Plans d'enseignement individualisés

En examinant les troisième et quatrième tableaux, on peut connaître le nombre et le pourcentage des répondants qui se sont prononcés sur les douze énoncés ainsi que leur position sur l'échelle de Likert. Si on fait fi du nombre de répondants, on remarque que ceux-ci accordent une très bonne note à l'ensemble des variables à expliquer. Seule la dernière au sujet du système éducatif ontarien en regard aux besoins des élèves, fait l'objet d'une forte dépréciation. À l'autre extrême, les enseignants sont nombreux à dire qu'ils consultent les PEI, que l'information que ces plans contiennent reflète le besoin des élèves, que les attentes sont pertinentes et qu'ils s'en servent. Mais ils sont tout aussi nombreux à dire que les normes du PEI devraient faire l'objet d'une révision. 
Yves Gauthier

\begin{tabular}{|l|l|l|l|l|l|l|}
\hline & $\begin{array}{l}\text { Utilise le } \\
\text { PEI }\end{array}$ & $\begin{array}{l}\text { Consulte le } \\
\text { PEI }\end{array}$ & $\begin{array}{l}\text { Attentes } \\
\text { sont } \\
\text { pertinentes }\end{array}$ & $\begin{array}{l}\text { Inf. reflète } \\
\text { le besoin } \\
\text { des élèves }\end{array}$ & $\begin{array}{l}\text { Outil péd. } \\
\text { utile }\end{array}$ & $\begin{array}{l}\text { Diagnostic } \\
\text { est précis }\end{array}$ \\
\hline 1 & $11(3,4 \%)$ & $11(3,4 \%)$ & $11 ; 3,4 \%$ & $8 ; 2,5 \%$ & $12 ; 3,7 \%$ & $8 ; 2,5 \%$ \\
\hline 2 & $26(8,1 \%)$ & $34(10,6 \%$ & $27 ; 8,4 \%$ & $21 ; 6,5 \%$ & $29 ; 8,9 \%$ & $23 ; 7,1 \%$ \\
\hline 3 & $62(19,3 \%$ & $43(13,4 \%$ & $60 ; 18,6 \%$ & $55 ; 17,0 \%$ & $76 ; 23,2 \%$ & $59 ; 18,2 \%$ \\
\hline 4 & $97 ; 30,1 \%$ & $90(28,0 \%$ & $94 ; 29,1 \%$ & $98 ; 30,2 \%$ & $106 ; 32,4 \%$ & $139 ; 42,8 \%$ \\
\hline 5 & $\begin{array}{l}126 ; \\
(39,1 \%)\end{array}$ & $\begin{array}{l}144 ; \\
(44,7 \%)\end{array}$ & $\begin{array}{l}131 ; \\
(40,6 \%)\end{array}$ & $\begin{array}{l}142 ; \\
(43,8 \%)\end{array}$ & $\begin{array}{l}104 ; \\
(31,8 \%)\end{array}$ & $\begin{array}{l}96 \\
(29,5 \%)\end{array}$ \\
\hline total & $322 ; 100 \%$ & $322 ; 100 \%$ & $322 ; 100 \%$ & $324 ; 100 \%$ & $\begin{array}{l}327 ; \\
(100 \%)\end{array}$ & $\begin{array}{l}325 ; \\
(100 \%)\end{array}$ \\
\hline
\end{tabular}

Tableau 3. Position du personnel-enseignant à l'égard des différents aspects du PEI

\begin{tabular}{|l|l|l|l|l|l|l|}
\hline & $\begin{array}{l}\text { Stratégies } \\
\text { sont } \\
\text { pratiques }\end{array}$ & $\begin{array}{l}\text { Connais- } \\
\text { sances } \\
\text { des } \\
\text { anomalies } \\
\text { sont } \\
\text { adéquates }\end{array}$ & $\begin{array}{l}\text { Revoir } \\
\text { les } \\
\text { normes } \\
\text { du PEI }\end{array}$ & $\begin{array}{l}\text { PEI est } \\
\text { un } \\
\text { outil } \\
\text { péd. }\end{array}$ & $\begin{array}{l}\text { PEI est une } \\
\text { tâche admin- } \\
\text { istrative }\end{array}$ & $\begin{array}{l}\text { Éducation } \\
\text { répond aux } \\
\text { besoins } \\
\text { des } \\
\text { élèves }\end{array}$ \\
\hline 1 & $\begin{array}{l}11 \\
(3,4 \%)\end{array}$ & $8(2,8 \%)$ & $8(2,6 \%)$ & $\begin{array}{l}16 \\
(4,9 \%)\end{array}$ & $23(7,1 \%)$ & $74(22,9 \%)$ \\
\hline 2 & $\begin{array}{l}33 \\
(10,3 \%)\end{array}$ & $29(8,9 \%)$ & $\begin{array}{l}21 \\
(6,8 \%)\end{array}$ & $\begin{array}{l}32 \\
(9,9 \%)\end{array}$ & $40(12,4 \%)$ & $99(30,7 \%)$ \\
\hline 3 & $\begin{array}{l}82 \\
(25,5 \%)\end{array}$ & $82(25,2 \%)$ & $\begin{array}{l}82 \\
(26,5 \%)\end{array}$ & $\begin{array}{l}93 \\
(28,7)\end{array}$ & $80(24,8 \%)$ & $96(29,7 \%)$ \\
\hline 4 & $\begin{array}{l}113 \\
(35,2 \%\end{array}$ & $136(41,8 \%)$ & $\begin{array}{l}85 \\
(27,4 \%)\end{array}$ & $\begin{array}{l}91 \\
(28, \%)\end{array}$ & $77(23,8 \%)$ & $40(12,4 \%)$ \\
\hline 5 & $\begin{array}{l}82 \\
(25,5 \%\end{array}$ & $70(21,5 \%)$ & $\begin{array}{l}114 \\
(36, \%)\end{array}$ & $\begin{array}{l}92 \\
(28,4 \%)\end{array}$ & $103(31,9 \%)$ & $14(4,3 \%)$ \\
\hline tot. & $\begin{array}{l}321 \\
(100 \%)\end{array}$ & $\begin{array}{l}325 \\
(100 \%)\end{array}$ & $\begin{array}{l}310 \\
(100 \%)\end{array}$ & $\begin{array}{l}324 \\
(100 \%)\end{array}$ & $323(100 \%)$ & $323(100 \%)$ \\
\hline
\end{tabular}

Tableau 4. Pertinence du PEI

Afin de déterminer s'il y a des différences significatives entre les opinions du personnel enseignant par rapport aux cinq variables indépendantes, c'est-à-dire l'âge, le sexe, le niveau d'enseignement, le nombre d'années d'expérience et le nombre de cours suivis, nous avons procédé à une analyse de variance (ANOVA). Les douze énoncés du questionnaire ont été soumis à cette analyse qui démontrent quelques différences significatives. 
Dans le premier énoncé du questionnaire concernant l'utilisation du PEI, l'analyse de variance ne démontre qu'une seule différence entre les opinions des enseignants et enseignantes. Cette différence s'explique par le niveau d'enseignement. Le personnel enseignant du niveau primaire-moyen utilise plus fréquemment le PEI et cette différence est significative $[\mathrm{F}=(2 ; 293)=3,724 ; \mathrm{p}>0,05]$. Aucune différence significative n'a été observée au sujet de la consultation du PEI et de la pertinence des attentes indiquées. Par contre, l'énoncé « l'information dans les PEI reflète les besoins des élèves » s'est avérée importante pour tous les répondants $[\mathrm{x}=4,06]$, et une différence significative a été observée entre les groupes $[\mathrm{F}=(2$; 271) $=4,440 ; p>0,05]$. Ce sont les répondants du cycle primairemoyen qui expliquent cette différence et ce sont eux qui ont suivi le plus de cours en enfance en difficulté: 72 au total comparativement à 28 pour ceux et celles du moyen-intermédiaire et 40 pour ceux de l'intermédiaire supérieur.

L'ensemble du personnel n'a pas une opinion très forte au sujet de l'utilité du PEI, [ $\mathrm{x}=3,80]$, mais les différences d'opinions sont tout de même significatives $[F=(2,312)=4,562 ; p>0,05]$. Cette variance est liée aux répondants du cycle primaire-moyen qui croient davantage à l'utilité du PEI. L'analyse de variance ne dénote aucune différence importante entre les répondants en regard à l'énoncé traitant de la précision du diagnostic de l'élève telle indiquée dans le PEI. Les stratégies d'enseignement proposées au personnel enseignant sont jugés plus ou moins favorablement $[x=3,69]$, et seul le cycle explique, une fois de plus, les différences significatives observées $[\mathrm{F}=$ $(2 ; 267)=3,282 ; \mathrm{p} \gg 0,05]$.

Les connaissances sur les anomalies des enfants en difficulté sont liées directement au nombre de cours suivis par les répondants et les différences sont significatives $[F=(3,212)=4,730 ; p>0,05]$. Il faut remarquer qu'il y a ici deux fois plus de répondants du cycle primaire-moyen qui détiennent la partie 3 de la qualification additionnelle du ministère de l'Éducation de l'Ontario. Plus de 63\% des répondants croient que le MÉFO devrait réviser les normes du plan d'enseignement individualisé, mais l'analyse de variance ne démontre aucune différence significative par rapport au niveau 
Yves Gauthier

d'enseignement.

Les répondants devaient aussi donner leur opinion sur le PEI, à savoir s'ils le voyaient comme un outil pédagogique et/ ou une tâche administrative. En examinant les données, nous pouvons conclure qu'il n'y a pas de lien entre l'attitude de ceux-ci face à ces deux énoncés. Tous les « Significatifs F » sont supérieurs au seuil de 0,05. Les moyennes (Voir tableaux 1 et 2) ne montrent pas de positions fortes sur l'échelle de 1 à 5 . Quant au dernier énoncé au sujet du système d'éducation en Ontario, la valeur du F $(3,129 ;>0,05)$ indique qu'il y a un lien significatif entre les répondants et le cycle d'enseignement. Les répondants du cycle primaire-moyen pensent que le système d'éducation répond favorablement à l'ensemble des besoins des élèves même si la grande majorité des autres répondants des autres cycles (Voir tableau 4) ne sont pas d'accord avec cet énoncé.

\section{Discussion}

Les résultats suggèrent que sur les douze énoncés, la moitié seulement est justifiée par les variables justificatives proposées. Nous constatons que deux des variables indépendantes, soit le sexe et le nombre d'années d'expérience, s'avèrent peu pertinentes en rapport avec l'opinion des répondants. En effet, les analyses démontrent un lien insuffisamment fort $(p>0,05)$ et, par conséquent, elles n'expliquent aucunement de façon significative les différences d'opinions exprimées par ceux et celles qui ont participé à la recherche. Par contre, la variable relative au cycle d'enseignement est celle qui émerge comme l'explication principale des six énoncés qui ont été jugés significativement favorables. Elle n'est dédoublée qu'une seule fois, dans l'énoncé relatif à la connaissance des anomalies, par le facteur portant sur la formation additionnelle en enfance en difficulté. Pour les propositions qui n'ont reçu aucune justification significative, il faudra chercher les explications ailleurs. L'une des explications possible réside dans la composition de l'échantillon. En effet, le personnel du cycle primaire-moyen est le plus représenté dans le groupe. Parmi tous les répondants, $47,5 \%$ enseignent au niveau

primaire-moyen, $21,9 \%$ sont au niveau moyen-intermédiaire et $26,1 \%$ 
sont dans les classes intermédiaire-supérieur. Les résultats obtenus traduiraient donc, dans une large mesure, l'opinion des enseignants du primaire-moyen; il n'en demeure pas moins cependant que ce sont eux qui s'inspirent davantage des PEI dans leur enseignement. Cette constatation n'est pas négligeable car l'intervention pédagogique est fortement encouragée dès que les difficultés sont dépistées au primaire-moyen puisque les chances de réussite de l'intervention sont plus élevées quand les enfants sont jeunes et qu'ils sont plus réceptifs aux modifications proposées dans leur enseignement.

Même si un lien significatif n'a pas été démontré que le PEI est considéré d'abord et avant tout comme une tâche administrative, la recherche laisse penser qu'elle va dans le sens des études de Bateman (1995) qui souligne que le PEI est vu par certains enseignants comme une charge de type bureaucratique. Aussi, en pensant à ce que les résultats montrent ici à propos des normes du PEI, par exemple, nous pouvons dire qu'ils confirment également les travaux de Thomson et Rowan (1995), lesquels attirent l'attention sur l'appréhension que le personnel enseignant ressent au sujet de la clarté des contenus du PEI. Sur le plan méthodologique, il importe de souligner que le personnel enseignant n'a été consulté qu'avec un seul outil d'enquête: le questionnaire. Les données issues du groupe focus n'ont pas été incorporées dans l'étude, mais ont plutôt servi à valider le questionnaire. Celui-ci comportait une partie « commentaires » qui a été peu exploitée par les répondants. Le questionnaire a connu un succès énorme et a permis de récolter des données empiriques qui ne sont pas toujours accessibles dans les études actuelles sur les PEI. Toutefois, bien que nous reconnaissions que l'usage d'un seul questionnaire suffit à comprendre de façon approfondie les représentations des enseignants, il suscite néanmoins des pistes intéressantes. Une triangulation des méthodes d'enquête serait nécessaire pour poursuivre les pistes ouvertes par cette recherche.

Enfin les résultats obtenus confirment l'intérêt de la théorie de la représentation sociale utilisée dans cette étude. L'hypothèse posait la prémisse qu'il existait un lien plus marqué entre les représentations que font les enseignants du PEI d'une part, et leur expérience scolaire et leur contexte de pratique, d'autre part. Nous mettons ainsi un doute sur l'influence déterminante des facteurs personnels comme l'âge ou 
le sexe sur les points d'ancrage des enseignants. L'œillère des représentations sociales que Doise et Palmonari (1986) font dériver des « rôles » et de « l'expérience » des acteurs sociaux revêt alors ici tout son sens. Elle nous aide à comprendre le lien entre certaines opinions exprimées à propos des $\mathrm{PEI}$ et le fait d'enseigner au primaire-moyen et de suivre des cours de perfectionnement en éducation de l'enfance en difficulté.

\section{Conclusion}

Les études actuelles sur les PEI ont tendance à projeter les désirs aussi compréhensibles soient-ils des auteurs à propos d'un PEI idéal, mais elles laissent dans l'ombre les représentations des enseignants qui sont pourtant un facteur critique dans la réussite de l'éducation des enfants en difficulté. La présente recherche a voulu souligner l'intérêt de comprendre cette réalité. En étudiant l'opinion du personnel enseignant à propos des $\mathrm{PEI}$, nous avons estimé que cette dernière s'exprime en lien avec l'ancrage des enseignants dans un contexte de pratique, qui, d'une manière ou d'une autre, colore leur point de vue. Le recours à l'enquête par questionnaire a permis de donner une assise empirique à cette manière d'aborder le regard des enseignants et enseignantes franco-ontariens. Les résultats obtenus sont éclairants et pourraient avantageusement servir de point de départ à des études utilisant des méthodes de recherche plus poussées, ces dernières pouvant ou non faire appel à une variété de méthodes. Les indications obtenues à propos du personnel enseignant du primaire-moyen nécessiteraient d'être mieux documentées en les replaçant, par exemple, dans le contexte particulier de ce cycle scolaire et dans celui, plus général, de la réforme scolaire en Ontario.

\section{Références}

Angelides, P., Charalambus, C., et Brasidas, V. (2004). Reflections on policy and practice of inclusive education in pre-primary schoolers in Cyprus. European Journal of Special Needs, 19(2), 211-13. 
Armstrong, P. (2003). Spaced out: Policy, difference and the challenge of inclusive education. Cluwer Academic Publishing.

Bateman, B. (1995). Writing Individualized Education Programs (IEPs) for Success. Dans Secondary Education and Beyond. Learning Disabilities Association.

Bateman, B. et Herr, C.M. (2003). Writing measurable IEP goals and objectives. Attainment Co. Inc.

Bateman, B., et Linden, M. (1998). Better IEPs: How to develop legally correct and educationally useful programs (3rd ed). Longmont, CO: Sopris West.

Côté, R., Pilon, W., Dufour, C., et Tremblay, M. (1990). Guide d'élaboration des plans de service et d'intervention. Québec: Groupe de recherche et d'études en déficience du développement, Département de psychopédagogie, Université Laval.

DiMaria, A. (1992). Art and the IEP. Dans F.E. Anderson et al. (dir). Art for all the children (213-218). Charles C. Thomas Publisher.

Doise,W. et Palmonari, A. (1986). L'étude des représentations sociales. Lausanne: Delachaux et Niestlé.

Drasgow, E., Yell, M.L., et Robinson, T.R. (2001). Developing legally correct and educationally appropriate IEPs. Remedial and Special Education, 22 (6), 359-73.

Gauthier, Y. (2004). Plan d'enseignement individualisé dans les écoles de l'Ontario: Analyse de cas des enfants surdoués et d'enfants en troubles d'apprentissage. Brock Journal of Education 13(2), 6-26.

Gauthier, Y. et Diallo, P. (2003). Enfance en difficulté en Ontario: Les initiatives provinciales dans le cadre de la réforme de l'éducation. Education Canada, 44 (1), 40-43.

Goupil, G. (1991). Le plan personnalisé en milieu scolaire. Boucherville: Gaëtan Morin Éditeur.

Goupil, G. (2004). Plans d'intervention et service et de transition. Boucherville: Gaëtan Morin Éditeur.

Goupil, G., Tassé, G., et Lawson, A. (1997). Plan de transition entre l'école et la vie adulte. Dépliant d'information. Montréal: Université du Québec à Montréal. Département de psychologie.

Hartwick, P.J., et Blattenberger, M.P. (1986). Taking the IEP home. A model for improved collaboration between residential and educational programs. Présentation faite à Denver. ERIC: ED 295390. 
Yves Gauthier

Haug, P. (2000). Words without deeds: Between special and inclusive education in Norway. Pedagogy, Culture and Society, 8(3), 291303.

Jodelet, D. (dir). (1989). Les représentations sociales. Paris: PUF.

Landry, A. (1990). Le plan d'intervention en milieu scolaire. Attitudes, 6, 7-11.

Ministère de l'éducation de l'Ontario (2004). Plan d'enseignement individualisé (PEI): Guide. (Internet: www.edu.gov.on.ca)

Mijer, C. J.W. (1998). Integration in Europe: Provision for pupils with special education needs. Trends in 14 European countries. Middelfart, Denmark: European agency for development in special needs education. ERIC: ED 426566.

Moscovici, S. (1989). Les représentations sociales. Paris: PUF

Moscovici, S. (1961). La psychanalyse et son image et son public. Paris: PUF.

Nutbrown, C. et Clough, P. (2004). Inclusion and exclusion in the early years: Conversations with European educators. European Journal of Special Needs Education, 19(3), 351-66.

Rapport d'évaluation. ERIC: ED 397580.

Revue française de pédagogie. Situations de handicaps et institution scolaire. No. 121 (numéro thématique).

Smith, S.W. et Kortering, L.J. (2001). Using computers to generate IEP process. Rethinking the process. Journal of Special Education Technology, 13(2), 107-16.

Smith, S.W. (2001). Involving parents in the IEP process. ERIC: ED 455658

Smith, S.W. (2000). Creating useful individualized programs (IEP) in special education. From intent to acquiescence. Exceptional Children, 57(1), 6-14.

Smith, S. W. (1990). Comparison of individualized education programs of students with behavioural and learning disabilities. Journal of Special Education, 24(1), 85-100.

Smith, S.W. et Simpson, R.L. (1989). An analysis of individualized education programs for students with behavioural disorders. Behavioural Disorders, 14(2), 197-216

Thomson, C. et Rowan C. (1995). Individual Education Plans in New Zealand Schools. Rapport d'évaluation. ERIC: ED 397580. 